Measurement and Theory in Disgust Sensitivity

\author{
Joshua M. Tybur \& Annika K. Karinen \\ Department of Experimental and Applied Psychology \\ VU Amsterdam
}

This pre-print corresponds with the following chapter: Tybur, J. M., \& Karinen, A. K. (2018). Measurement and theory in disgust sensitivity. In Zeigler-Hill, V., \& Shackelford, T. K. (Eds.) Handbook of Personality and Individual Differences, 159-179. 


\section{Measurement and Theory in Disgust Sensitivity}

Surveys conducted in the United States show that people report disgust toward actions and objects that inhabit some of the most important corners of our lives. We are disgusted by the prospect of eating certain foods, the sights and smells of other people's bodies, the thought of sexual contact with most of the people on earth, and considerations of others' moral shortcomings (Haidt et al., 1994; Tybur et al., 2009). International surveys indicate that disgust's relevance to food choice, mating, and morality is not a quirk of US culture (Curtis and Biran, 2001; Haidt et al., 1997; cf. Kollareth and Russell, in 2017). Despite disgust's far-reaching consequences, only scarce work was devoted to understanding the emotion through most of the twentieth century (Rozin et al., 2009). In the 1990s, however, the dearth of research to the topic caught the attention of a handful of scientists, who described disgust as both the 'forgotten emotion of psychiatry' (Phillips et al., 1998) and as a scarcely investigated - yet key - cog in the mechanisms that underlie our moral psychology (Haidt et al., 1993; Haidt et al., 1997). Calls for greater interest in disgust were heard across the behavioral sciences, with researchers using the science of disgust to better understand political attitudes (Inbar et al., 2012), food preferences (Fessler et al., 2003), health behavior (Reynolds et al., 2014), personality (Tybur and De Vries, 2013), psychopathology (Olatunji and Sawchuk, 2005), aggression (Pond et al., 2012), and moral judgments (Chapman and Anderson, 2013, 2014).

Although much of this work on disgust has focused on understanding the psychological processes underlying disgust and their resulting effects on behavior (e.g., Tybur and Lieberman, 2016), the majority of disgust research has focused on individual differences. Largely using selfreport instruments, researchers have tested how disgust sensitivity (DS) - that is, reported intensity of disgust toward the types of things that arouse at least a little disgust in most healthy 
adults - relates to the variety of topics detailed earlier. Results from these studies portray the disgust sensitive individual as someone who is averse toward new experiences (Tybur and De Vries, 2013), politically conservative (Inbar et al., 2012), prone to moral judgment (Chapman and Anderson, 2014), and more likely to have anxiety disorders (Olatunji and Sawchuk, 2005). Relative to the prodigious measurement of DS, though, little work has critically analyzed the validity of the multiple DS instruments used in the literature and the nature of DS as a construct. This chapter aims to provide a survey of the DS literature and, in doing so, comment on the dimensionality of DS, the mechanistic roots of DS, the developmental origins of DS, and a theoretical framework that can organize the literature.

\section{Progression of DS Measurement}

If you think about your friends and family members, you can probably pick out some individuals who seem to experience disgust more intensely or frequently than others - some people who are more bothered by disgusting odors, who hesitate more to drink out of the same cup as a friend, or who blanch at the thought of using a portable toilet. Even if this variation is apparent (as we suspect), it is not necessarily well captured by the lexical approach that has come to dominate the development of personality models (Goldberg, 1990). Of course, disgust need not be unique in this sense since lexical studies typically remove terms describing emotional states (e.g., 'angry,' 'disgusted') from their collection of trait adjectives (Allport and Odbert, 1936). Nevertheless, tendencies to experience some emotions are well captured by the lexical method. Consider proclivity to become angry, which is described by enough adjectives (e.g., 'hotheaded,' 'short-fused,' 'touchy,' 'quarrelsome') to form its own facet within the Big Five Emotional Stability factor (Anger) and the Five Factor Model Neuroticism factor (Hostility). In contrast, we can only think of one English term - squeamish - that (imperfectly) describes 
disgust at a trait level. An informal survey among our international colleagues suggests that Dutch, German, French, Spanish, Turkish, Chinese, Finnish, Greek, and Romanian similarly have a meager number of words that describe DS. Consequently, no personality facet seems to describe DS, and DS has remained largely invisible to a psychological community that, until recently, seemed largely unconcerned with understanding disgust (Rozin et al., 2009).

The first development of a DS instrument thus occurred outside the personality domain when Rozin et al. (1984) aimed to test for within-family similarity in food attitudes. Based on an earlier developed taxonomy of food attitude determinants (Rozin and Fallon, 1980), Rozin and colleagues (1984) suggested that disgust shapes food acceptance versus rejection, and that food attitudes are influenced by what they interchangeably labeled as 'contamination sensitivity' and 'disgust sensitivity.' Given the absence of DS instruments in the literature, they went on to generate items intended to capture this variability, with example items including 'How much would you like to eat soup from a thoroughly washed used dog bowl,' and 'How much would you like to eat a cookie after a bite had been taken by a waiter in a restaurant.' With this, the first DS instrument was born. It was not widely adopted in the literature, though, and its validity was scarcely investigated. Further, as Haidt et al. (1994) later pointed out, the instrument's item content exclusively concerned food, which is only one of many potential categories of disgust elicitors. Haidt and colleagues (1994) therefore set out to develop an instrument that would better represent the variety of disgust elicitors and, perhaps, map out the dimensionality of DS. This project led to the development of the Disgust Scale, which would be used widely in the social, clinical, and personality psychology literature. 
Several other DS instruments were developed after the Disgust Scale, including the Disgust Emotion Scale (Walls and Kleinknect, 1996), the Disgust Propensity and Sensitivity Scale (Van Overveld et al, 2006 - see Box 1), the Three Domain Disgust Scale (Tybur et al., 2009), and revised versions of the Disgust Scale (e.g., Olatunji, Williams et al., 2007). We will provide a detailed summary of the development and validation of the Disgust Scale-Revised (DS-R) and the Three Domain Disgust Scale (TDDS) since these two instruments have been most widely administered and most widely used to make inferences about the dimensionality of DS.

\section{The Disgust Scale}

Before developing the Disgust Scale, Haidt and colleagues (1994) first attempted to identify the categories (or domains) that represent disgust elicitors. To do so, they asked 20 respondents to describe their three most disgusting experiences, and then to list as many disgusting things as they could think of. The authors reviewed the 221 descriptions and interpreted them as representing eight domains - food, sex, body products, body envelope violations (e.g., wounds), moral violations, animals, hygiene, and death. This interpretation would go on to shape how the field interpreted the dimensionality of disgust sensitivity for decades (see Box 2).

After writing a large pool of items intended to reflect these eight domains, the authors asked a sample of undergraduates and local employees to rate the items. Based on the results of this initial survey, they decided to eliminate all moral items from the scale because those items had noticeably lower item-total correlations than the other items. After further item parsing, they retained 28 items for their scale - four for each of their targeted domains. Finally, they decided to add another four items intended to assess variability in magical thinking (contagion). This 
process led to the final version of the Disgust Scale, a 32-item measure with eight labeled domains (see Figure 60.1). For half of the items, respondents indicated true (1) or false (0) (e.g., 'I think it is immoral for people to seek sexual pleasure from animals'); for the other half of the items, respondents indicated not 'not disgusting at all' (0), 'slightly disgusting' ( 0.5$)$, or 'very disgusting' (1; e.g., 'A friend of yours offers you a piece of chocolate shaped like dog-doo').

\section{-- BOX 2 HERE --}

Although widely used for over a decade, the Disgust Scale contained measurement shortcomings that compromised its validity as a measure of DS. Most critically, factor analyses indicated that the eight factor structure proposed by Haidt and colleagues (1994) did not accurately represent the scale's dimensionality. That is, no evidence supported the idea that, for example, individual differences in disgust toward food are distinct from individual differences in disgust toward animals. Nevertheless, researchers often made inferences based on assumptions that subscales measured distinct constructs (e.g., Goldenberg et al., 2001; Rozin et al., 2005). Further, as would be expected given the small number of items (four) per domain, the subscales had low reliabilities (Cronbach's alphas generally below 0.60 ), which varied across putative domains. Hence, even if the subscale composites did accurately parse the dimensionality of the scale, they were substantially composed of error variance.

With these issues in mind, Olatunji and colleagues (2007) more thoroughly examined the scale's factor structure with the goal of revising the instrument. After factor analyzing scale items on a large sample of undergraduates $(\mathrm{N}=655)$, the authors interpreted a parallel analysis as indicating the presence of three factors. They then removed seven items (including all four sex items) that either did not load strongly on these factors or had correlated error terms (as assessed by subsequent confirmatory factor analysis). This process led to the Disgust Scale-Revised (DS- 
R), which includes 25 of the Disgust Scale's original 32 items. The three DS-R factors were labeled Core Disgust (e.g., 'It bothers me to hear someone clear a throat full of mucus'), Animal Reminder Disgust (e.g., 'Your friend's pet cat dies and you have to pick up the dead body with your bare hands'), and Contamination Disgust (e.g., 'I never let any part of my body touch the toilet seat in a public washroom').

Despite arguments that these subscales measure different constructs (Olatunji et al., 2007 ; Olatunji et al., 2008), researchers often sum items across factors, with the resulting composite interpreted as reflecting 'total' trait disgust (e.g., Chapman and Anderson, 2014; Olatunji et al., 2013; Sherman et al., 2012). This strategy seems reasonable in light of the high correlations between DS-R factors. Using latent variable estimates, Olatunji and colleagues (2007) report that the Core and Animal Reminder factors correlate $r=0.84$, that the Core and Contamination factors correlate $r=0.79$, and that the Animal Reminder and Contamination factors correlate $r=$ 0.66. That said, interpretations of this higher order factor as a total or general factor that subsumes all dimensions of DS sit uneasily with observations made during the development of the Disgust Scale. Recall that Haidt and colleagues (1994) included socio-moral items in their preliminary version of the Disgust Scale, since such items were nominated during their item generation process, but later removed those items based on their low item-total correlations. These low item-total correlations were interpreted as problematic, but they could suggest that those items were tapping a construct distinct from that measured by the other DS items. Recall further that, in developing the DS-R, Olatunji and colleagues (2007) removed all sexual items from the Disgust Scale. Although eliminating sexual and moral items produced a more homogenous DS measure, later evidence would suggest that the DS-R does not assess a general tendency to be disgusted, but rather a tendency to experience one type of disgust. 


\section{The Three Domain Disgust Scale}

Noting the exclusion of sexual and moral items from the DS-R, Tybur and colleagues (2009) developed the TDDS using an approach similar to that which produced the Disgust Scale, with two exceptions. First, whereas sexual and moral items were removed from the Disgust Scale and the DS-R based on their weak covariances with items describing cues to pathogens, they were retained and subjected to factor analysis during the development of the TDDS. Second, whereas Disgust Scale items were written by the scale developers using what has been described as 'weird' phrasing (i.e., generating scenarios that are uncommon and potentially difficult to relate to; see Gray and Keeney, 2015), which might introduce systematic method variance, TDDS items were generated from a panel and were selected partially based on their expected relatability to participants. After gathering 105 disgust-eliciting items from 14 individuals, Tybur and colleagues eliminated items that were redundant, were expected to have little variability, or described items or individuals idiosyncratic to the sample's local environment. This led to a pool of 58 items, which were rated for disgust by a sample of 160 students. A further 10 items were removed due to high skewness or kurtosis, and the remaining 48 items were factor analyzed in this sample and another sample of 300 students. Exploratory factor analyses on both samples indicated the presence of three factors, the first of which appeared to reflect item content similar to that from the DS-R (and was dubbed a 'pathogen' factor), and the second and third of which appeared to reflect sexual and moral item content, respectively. The final version of the TDDS contained 21 items (seven for each factor), which were retained based on high factor loadings, low error covariances between items, and enough items for subscale composites to have reliabilities around $\alpha=0.80$. Pathogen items include 'Stepping on dog poop' and 'Standing close to a person who has body odor,' sexual items include 'Finding out that someone you don't like 
has sexual fantasies about you' and 'Hearing two strangers having sex,' and moral items include 'Deceiving a friend' and 'A student cheating to get good grades.' All items are answered on a 0 (not at all disgusting) to 6 (extremely disgusting) response scale.

\section{-- FIGURE 1 HERE --}

The TDDS pathogen subscale largely corresponds with the DS-R total score, with one study of 353 participants reporting a high correlation between the two, $r=0.65$ (and $r=0.76$ when disattenuating for unreliability; Tybur et al., 2010). The sexual and moral subscales are only modestly related to the DS-R, however, with the same study reporting correlations of $r=$ 0.38 and $r=0.12$, respectively $(r \mathrm{~s}=0.44$ and 0.14 , respectively, when disattenuating for unreliability). In the remainder of this chapter, we will therefore describe disgust sensitivity along these three domains: pathogen, sexual, and moral. Some of the pathogen disgust findings we report will be based on the Disgust Scale, others on the DS-R, and others on the pathogen domain of the TDDS. Any findings concerning sexual or moral disgust sensitivity are based on the TDDS.

\section{Interpreting Disgust Sensitivity Measures}

In developing the Disgust Scale, Haidt and colleagues (1994) noted that there were, at the time, no validated measures of DS. This statement was true. But was the absence of a DS instrument problematic? As discussed earlier, adjectives for describing DS are short in number, so DS might not be well represented in personality space based on lexical approaches. Even so, those who tend to experience disgust might be the same individuals that tend to experience other negative emotions - that is, DS might be a manifestation of neuroticism or emotionality, and any conclusions based on DS instruments might be better attributed to higher order personality factors. Further, does DS relate to other signatures of disgust, such as avoidance behavior or 
physiological responses to cues to pathogens? Or does it only measure some sort of selfpresentational tendencies? Personality, physiological, and behavioral studies can answer these types of questions, and they can provide us with critical information for interpreting DS instruments.

\section{DS and Neuroticism/Emotionality}

Some early studies suggested that pathogen DS might indeed be a manifestation of neuroticism. For example, one study of 132 undergraduates reported that pathogen disgust correlates strongly with NEOPI-R neuroticism, $r=0.45$ (Druschel and Sherman, 1999 ). A later study using the DS-R reported a similarly strong correlation with neuroticism as measured by the NEOFFI among a sample of 247 undergraduates, $r=0.46$ (Olatunji et al., 2008). Other evidence suggests that the relation between pathogen DS and neuroticism is less strong. In a large student sample $(\mathrm{N}=477)$, pathogen DS was only weakly correlated with NEO-PI-3 neuroticism, $r=0.10$ (Tybur et al., 2011). In a similarly sized sample of the general Dutch population $(\mathrm{N}=476)$, pathogen DS was again weakly related to 5DPT neuroticism, $r=0.13$, and to HEXACOPI-R emotionality, $r=0.18$ (Tybur and De Vries, 2013). These weaker relations with neuroticism and emotionality are consistent with other research reporting that neuroticism is unrelated to both self-reports of disgust toward films depicting mutilated animals and food being spit on (Hennig et al., 1996) and toward eye-blink startle responses to disgusting film clips (Wilson et al., 2000). Why then do some studies report such strong relations between neuroticism and DS? The stronger of the relations described earlier used the Disgust Scale or the DS-R to measure pathogen DS. This instrument, while strongly correlated with the pathogen domain of the TDDS, contains many items that ask participants how 'bothered' or 'upset' they would be in situations described in items (e.g., 'It bothers me to hear someone clear a throat full 
of mucous'). Hence, features of the scale's item content apart from disgust might relate to neuroticism.

The relations between neuroticism/emotionality and sexual DS and moral DS are similarly modest. Two of the studies described earlier also found that sexual DS is largely unrelated to NEOPI-3 and 5DPT neuroticism and HEXACO emotionality, $r s=0.03,0.19$, and 0.10 , respectively, and that moral DS is, if anything, even more weakly related to the same personality measures, $r=-0.13,-0.03$, and 0.02 (Tybur et al., 2011; Tybur and De Vries, 2013). In sum, personality studies suggest that DS is not an epiphenomenon of a tendency to experience negative emotions. Further, as we discuss next, DS is only weakly to moderately related to other personality dimensions. Nevertheless, personality data can give us some insight into what types of traits bundle together with DS, and hence how we should interpret DS as a construct. We base our overview of personality and DS on the two large samples that have administered the NEO PI-3 and HEXACO-PI-R (Tybur et al., 2011; Tybur and De Vries, 2013; see Table 1 for a summary).

\section{DS and Other Personality Factors}

Of the NEO factors, pathogen DS relates most strongly (negatively) to openness to experience, $r=-0.24$, with correlations roughly equal across facets. The correlation with HEXACO openness was also negative and non-zero, though weaker in magnitude, $r=-0.11$. These relations might reflect the fact that some unconventional experiences - especially those related to novel foods, hygiene customs, and physical contact (Schaller and Murray, 2008) - can elicit pathogen disgust, and individuals who are more easily disgusted by pathogen cues might thus avoid those types of situations. Interestingly, though, the relation between HEXACO agreeableness and pathogen DS is directionally stronger, with higher pathogen DS associated 
with lower agreeableness, $r=-0.17$. Pathogen DS is similarly related to NEO agreeableness, $r=$ -0.13 , and its strongest facet-level relation is with trust, $r=-0.16$. This finding resonates with another series of studies reporting that pathogen DS relates negatively to generalized social trust independent of agreeableness (Aarøe et al., 2016 - although we note that these studies assessed agreeableness using short-form measures, which can produce erroneous conclusions that relations between other variables - such as DS and trust - exist independent of personality; see Credé et al., 2012; De Vries, 2013). These relations could reflect higher pathogen DS individuals being less open to potentially pathogenic contact with others. However, this interpretation might also predict a negative relation between pathogen DS and extraversion, but no such relation is observed in NEO or HEXACO data, $r \mathrm{~s}=-0.05$ and 0.01 , respectively. Further research can clarify how to interpret relations between pathogen DS, agreeableness, and generalized social trust (and, further, openness to experience).

Like pathogen DS, sexual DS relates negatively to NEO openness, $r=-0.38$. Unlike pathogen DS, though, this relation appears to vary across openness facets, with some facets relating modestly to sexual DS (e.g., aesthetics, $r=-0.20$, and feelings, $r=-0.18$ ), and one facet - values, which especially relates to liberal versus conservative political ideology - having a particularly strong relation, $r=-0.42$. Further, sexual DS was unrelated to HEXACO openness, even though openness is practically identical across HEXACO and NEO instruments (Ashton and Lee, 2009). These inconsistencies suggest (a) that relations between sexual DS and openness might be a byproduct of the relation between sexual DS and political conservatism - a topic we will return to later in the chapter - and, relatedly, that (b) these relations might vary across societies as a function of degree to which political identification relates to sexual attitudes (i.e., US versus Dutch political systems; recall that the NEO study was conducted using a US 
undergraduate sample, and the HEXACO study was conducted using a Dutch sample varied in age and education). Sexual DS only significantly related to one other NEO factor: agreeableness, $r=0.18$. This relation also did not generalize to the HEXACO, although the lack of correspondence might reflect differences between NEO and HEXACO agreeableness rather than differences between US and Dutch societies. Whereas NEO agreeableness reflects not only tendencies to be kind, gentle, flexible, and forgiving, but also tendencies to be honest and modest, the HEXACO model treats honesty-humility as a separate factor with distinct construct validity (Ashton et al., 2014). Sexual DS did relate to HEXACO honesty-humility, $r=0.17$, and, of the NEO agreeableness facets, those most strongly related to honesty-humility (straightforwardness and modesty, $r \mathrm{~s}=0.20$ and 0.16 , respectively) were most strongly related to sexual DS. Together, these patterns suggest that low sexual DS partially reflects an orientation toward behaving in self-enhancing and exploitative manners.

Moral DS mirrors sexual DS in its disjunctive relation with NEO versus HEXACO agreeableness. Like sexual DS, moral DS relates to NEO agreeableness, $r=0.25$, and especially those facets related to honesty-humility (straightforwardness, altruism, and modesty, $r \mathrm{~s}=0.27$, 0.22 , and 0.18 , respectively). And, while it is unrelated to HEXACO agreeableness, $r=0.07$, it is related to HEXACO honesty-humility, $r=0.29$. As with sexual DS, these findings suggest that individuals who behave more honestly, straightforwardly, and humbly score higher on moral DS. Unlike sexual DS, though, moral DS is moderately related to conscientiousness, as assessed by both NEO and HEXACO instruments, $r \mathrm{~s}=0.28$ and 0.26 , respectively.

The varied and weak-to-moderate relations between domains of DS and personality provide two key pieces of information. First - and in concert with factor analyses on the TDDS they suggest that DS is not a uni-dimensional construct, and references to 'total' or 'general' DS 
overlook potentially important differences between DS dimensions. Second, they tell us that DS is not merely a manifestation of a higher order personality dimension. Nevertheless, the patterns of relations with personality can offer a data-driven starting point for generating hypotheses for how to interpret DS and, potentially, to explain why individuals vary in DS. We will propose such explanations later in the chapter. First, though, we will describe strong relations between DS domains and other constructs - relations that can further inform how we interpret DS.

\section{Pathogen DS and Perceived Vulnerability to Disease}

In contrast with its modest relations with personality factors, pathogen DS relates strongly to germ aversion factor of the Perceived Vulnerability to Disease (PVD) scale (Duncan et al., 2009), which assesses comfort with infection-risky social events (e.g., 'It really bothers me when people sneeze without covering their mouths'), with correlations reported across three studies as $r=0.55, r=0.51$, and $r=0.55(\mathrm{Ns}=983,214$, and 254, respectively; Duncan et al., 2009; Tybur et al., 2015 a). This strong relation is specific to pathogen DS, with sexual DS and moral DS more modestly related to germ aversion ( $r s=0.31$ and 0.07 ; Tybur et al., 2015 a). Based on the strong relation between germ aversion and pathogen DS, both are often used interchangeably as measures of pathogen-avoidance motivations (or, as measuring trait level behavioral immune system variation - see Tybur et al., 2014). That said, little theory and data have aimed to test whether and how the constructs differ. Lack of perfect overlap could reflect measurement differences (e.g., scale anchor differences across the instruments). Or it could indicate that, while both constructs relate strongly to a higher order pathogen-avoidance factor, they reflect different types of motives. For example, Gangestad and Grebe (2014) have argued that variation in avoidance of human-transmitted pathogens might be unique from avoidance of other pathogens, given the unique costs of avoiding social contact (cf. Park, 2015). Of course, 
further research is necessary to tease apart potential differences between germ aversion and pathogen DS.

\section{Sexual DS and Sociosexuality}

The relation between sexual DS and sociosexual orientation (i.e., openness to sex outside of a committed relation) mirrors that between pathogen DS and germ aversion, both in terms of magnitude and proposed conceptual equivalence. One study found that sociosexual attitudes (e.g., 'I can imagine myself being comfortable and enjoying "casual" sex with different partners') correlate strongly (negatively) with sexual DS, $r=-0.54$, but not with moral or pathogen DS (rs $=-0.10$ and -0.16 , respectively; Tybur et al., 2015 a). Another study that assessed a broader array of sociosexuality - including attitudes, desires, and past behavior found correlations of a similar magnitude (Al-Shawaf et al., 2015). Further, sexual DS and sociosexuality relate similarly to other variables, including socio-political conservatism ( $\mathrm{rs}=$ 0.26 versus -0.23 , respectively; Tybur et al., 2015a) and HEXACO emotionality ( $\mathrm{rs}=0.31$ versus -0.24 , respectively) and honesty-humility ( $\mathrm{rs}=0.31$ versus -0.36 , respectively; Bourdage et al., 2007; Tybur and De Vries, 2013). Notably, relations between sociosexuality and Dark Triad personality traits mirror those between sexual DS and honesty-humility, and they suggest that anti-social personality clusters facilitate a short-term mating strategy (Jonason et al., 2009).

Based on the strong correlations between sexual DS and sociosexuality, multiple studies have treated both variables as assessing the same sexual strategies construct; namely, openness to and pursuit of sexual behaviors outside of intercourse in a monogamous relation (Kurzban et al., 2010; Quintelier et al., 2013; Tybur et al., 2015 a). Such interpretations run counter to arguments and data presented by Simpson and Gangestad (1991), who suggested that sociosexual orientation is distinct from a number of other sexual variables, including sex drive, 
sexual prudishness, and, especially pertinent here, sexual disgust. As with germ aversion and pathogen DS, further research is needed to better understand how sociosexuality and sexual DS differ from each other and from a broader array of sexual traits.

Up to here, our discussion of DS validity has considered only relations between self-report instruments. These relations provide some hints at what DS is and what it is not. However, they do not speak strongly to DS instruments actually reflecting intensity of disgust responses to typical disgust elicitors. Another set of findings - those using behavioral data - can speak to this issue.

\section{Behavioral Validations of DS}

As its name implies, pathogen disgust is associated with motivations that appear tailored to keeping pathogens at bay. Consider the canonical disgust face, which is characterized by (1) a closing of the eyes and, a lowering of the eyebrows, both of which reduce the exposed surface area of the eyes, (2) a wrinkling of the nose, which reduces air intake, and (3) a lowering of the lips, which reduces the probability of objects entering the mouth (or, alternatively, if something is already in the mouth, a protruding tongue, which expels the contents of the mouth; Susskind et al., 2008). Each of these actions partially seals off an entryway through which pathogens can enter the body. Behaviors apart from facial expression also appear specialized for neutralizing pathogens. Disgust is associated with motivations to avoid physical contact with the disgust elicitor - physical contact that would allow pathogens to be transmitted from disgust elicitor to human (Hertenstein et al., 2006; Roseman et al., 1994). Does pathogen DS, as assessed by selfreport instruments, relate to these types of pathogen-neutralizing behaviors?

Multiple studies employing behavioral avoidance tasks (BAT) suggest that it does. In BATs, researchers record whether participants are willing to physically contact an object, and 
what degree of contact they will engage in. For example, in one study, participants were presented with a cookie on the floor and were asked to (a) hold the cookie, (b) touch the cookie with their lips, and (c) eat the cookie (Deacon and Olatunji, 2007). Similar progressions were used for a used hair comb and a bedpan filled with toilet water. Pathogen DS (negatively) predicted the number of steps completed in the tasks, even when controlling for participant sex, anxiety, and depression. Similar results have been obtained for BATs in which participants were asked to touch tissues used by someone who had the common cold (Fan and Olatunji, 2013); touch a sterilized cockroach (Rozin et al., 1999); touch a colonoscopy bag (Reynolds et al., 2014); and touch moldy fruit (Olatunji, Lohr et al., 2007). In contrast, pathogen DS does not predict avoidance of watching or committing socio-moral violations (Van Overveld et al., 2010), and sexual DS and moral DS do not predict avoidance of contact with sinks, trash cans, and toilets in a public restroom (Olatunji et al., 2012). Similarly, pathogen DS - but not sexual DS or moral DS - relates to galvanic skin response to images of pathogen cues (Olatunji et al., 2012).

Only a few studies have tested whether DS relates to facial responses to disgust-eliciting stimuli. In one study of 47 participants, facial electromyography (EMG) indicated that pathogen DS was unrelated to the degree of levator labii (a key muscle in the disgust facial response) activation in response to disgust-eliciting images (Stark et al., 2005). In another study of 60 participants, EMG again indicated that pathogen DS was unrelated to levator labii activation in response to a disgust-eliciting film clip (De Jong et al., 2011). Of course, these studies are not well powered to detect small relations between DS and facial responses - they only had $28 \%$ and $34 \%$ power to detect a correlation of $r=0.25$. Nevertheless, they hint at two interesting possibilities. First, they could suggest that variability in some anti-pathogen responses, including subjective feelings of disgust and physical avoidance, is distinct from variability in other anti- 
pathogen responses, such as reducing the degree to which the eyes, nose, and mouth are exposed to pathogens. Second (and, perhaps, alternatively), they could suggest that variability in facial response to disgust elicitors reflects variability in motivations to communicate the presence of pathogens to others (see Fridlund, 1991). Once again, further research is needed to adjudicate between these possibilities.

The majority of studies testing how DS relates to behavior have presented participants with cues to pathogens. One exception examined how DS relates to aggression. Reasoning that disgust motivates avoidance - and that aggression involves approach-oriented motivations (Harmon-Jones and Peterson, 2008) - Pond and colleagues (2012) suggested that DS should relate negatively to aggression. They found that participants higher in moral DS and in sexual DS - but not pathogen DS - delivered fewer high intensity noise-blasts in a behavioral aggression paradigm (notably, though, this study did not report unique effects of moral DS vs. sexual DS, and it did not control for participant sex - a variable strongly related to both aggression and sexual DS). That said, we are unaware of any studies that have examined how sexual DS relates to behavioral responses to unwanted sexual advances or how moral DS relates to behavioral responses to individuals who have committed moral transgressions. Naturally, such studies present ethical challenges that surpass asking participants to touch tissues or sterilized cockroaches. Nevertheless, they would greatly improve our interpretation of sexual DS and moral DS.

In sum, findings gleaned from a variety of methods - including self-report instruments, behavioral avoidance tasks, and physiological measures - provide the groundwork for how we should interpret DS. However, a theoretical framework is required to integrate these empirical findings and transform this groundwork into a firm foundation. In the next section, we will extend 
a theoretical framework for understanding the experience of disgust (Tybur et al., 2013) to understanding variability in DS.

\section{The Tradeoffs of Disgust}

Although pathogen disgust can steer individuals away from physical contact with pathogens, it is an imperfect anti-pathogen defense. Infectious microorganisms are invisible to the naked eye, and so people do not have perfect knowledge of the infection consequences of a behavior. Indeed, humans were unaware of the existence of bacteria until Antonie van Leeuwenhoek first used a microscope to peer into the world of microbes in the late seventeenth century. Any evolved anti-pathogen psychological mechanisms, including those underlying disgust, must thus be designed to detect pathogens based on cues that we can detect - things like the colors, smells, sounds, and textures associated with pathogens, that is, they must use sensory properties that are probabilistically (but imperfectly) associated with pathogens. Such systems sometimes - perhaps often - produce false-alarms (detects pathogens when none are present) and misses (fails to detect pathogens when they are present). Further complexities arise when we consider that some beneficial behaviors increase the probability of contact with pathogens. Consider eating, which can transport pathogens resting on the hands or in food into the body. Avoiding eating altogether, while reducing the probability of infection, would lead to starvation. Similarly, physical contact with friends and offspring and sexual contact with mates, while potentially infectious, are each necessary for survival and reproduction.

Experiencing pathogen disgust can thus be viewed as the outcome of a non-conscious tradeoff between the probabilistic (based on cues associated with pathogens) infection costs and the other (e.g., sexual, nutritional, social) probabilistic benefits of physical contact (Tybur and Lieberman, 2016). This tradeoff can vary across contexts. When calories are needed, people 
might experience less disgust toward foods with the textures, colors, or smells indicative of bacterial presence (Hoefling et al., 2009). When another individual is perceived as having sufficient sexual quality or compatibility, a person might be willing (and, perhaps, eager) to engage in potentially infectious behaviors, such as putting his or her tongue in this other individual's mouth. Indeed, one study even finds that women who are sexually aroused lower their disgust thresholds in non-sexual activities (e.g., touching toilets; Borg and De Jong, 2012). When feces come from one's own baby, people experience less disgust relative to when it comes from another person's baby (Case et al., 2006). Although experiences of sexual disgust and moral disgust correspond with costs and benefits distinct from those associated with pathogen disgust, they likely arise from psychological mechanisms that are similarly designed to make tradeoffs (Tybur et al., 2013).

This same perspective can frame how we think about trait-like DS. Individuals with higher pathogen DS, sexual DS, and moral DS ultimately benefit from experiencing more intense disgust in each domain (e.g., through avoiding contact with pathogens, costly sexual interactions, inaction in response to moral violations), but they also pay costs for this disgust. Higher pathogen DS might lead people to avoid foods that are nutritious, offend social allies by refusing physical contact (e.g., with a sweaty palm), and expend time and energy in proximal avoidance of potentially pathogenic objects; higher sexual DS might lead people to avoid beneficial sexual partners and behaviors; and higher moral DS might lead people to avoid cooperative interactions with or express condemnation toward others who would actually be valuable social partners or who would counter-aggress. These costs and benefits can impact individuals differently. Consider, for example, the costs of rejecting nutritious food for people who are chronically nutritionally stressed vs. for people who have access to plentiful foods. Higher pathogen DS 
would be more beneficial for the latter individuals than for the former, since they can 'afford' to only accept foods assessed as being unlikely to house pathogens. And, just as context-specific disgust follows an adaptive logic (e.g., do not experience pathogen disgust when contact value is high, such as when handling your own baby), we suspect that DS is in some sense strategic, with individuals calibrating their DS based on a non-conscious weighting of costs and benefits (see Tybur and Lieberman, 2016, and Gangestad and Grebe, 2014, for further discussions).

Thinking in terms of the three dimensions of DS and their distinct underlying strategic tradeoffs can provide a fruitful framework for interpreting relations between DS and agreeableness. Recall the negative relation between pathogen DS and openness to experience and, specifically, trust (Aarøe et al., 2016; Tybur and De Vries, 2013; Tybur et al., 2011; see Table 60.1). The relation can be interpreted as suggesting that individuals who anticipate fewer benefits from social contact (e.g., based on perceptions of others' lack of trustworthiness) have less to lose from being disgusted by others, and so they more strongly prioritize the antipathogen benefits of higher pathogen DS. Recall further the positive relation between conscientiousness and moral DS. Here, individuals who are harder working and more diligent have more to lose when others betray, lie, cheat, steal, and freeload, and so they are more readily disgusted by such moral violations. Of course, these interpretations should be treated as tentative. Nevertheless, they can be used to generate and test competing hypotheses that can be used to reject, refine, or verify such interpretations. We now provide two examples of how considerations of disgust's distinct functions and dimensionality have proven useful.

\section{DS and Political Ideology}

Over the past ten years, several studies found that in the United States people with more politically conservative views have higher pathogen DS than people with more politically liberal 
views (Inbar and Pizarro, 2016; Terrizzi et al., 2013). This relation has widely been interpreted using one of the foundational tenets of the parasite stress theory of sociality (Fincher and Thornhill, 2012): that people develop immunity to the pathogens within their local ecologies but not to pathogens in foreign ecologies - and so contact with a member of a foreign ecology poses a greater infection risk than contact with an individual of a native ecology. Given that intergroup bias is a hallmark of conservative ideology, people who allocate more resources in avoiding pathogens find the putatively pathogen-neutralizing aspects of conservative values appealing (see Tybur et al., 2010, for an overview of this perspective).

Considerations of the dimensionality of both DS and ideology have encouraged alternative interpretations of the relation between DS and politics. Multiple studies have reported that pathogen DS is unrelated to ideology when controlling for sexual DS (Tybur et al., 2010) and, further, that sexual DS and sociosexuality partially or fully mediate the relations between multiple measures of pathogen avoidance and multiple measures of ideology (Tybur et al., 2015a; see also Shook et al., 2015 and Tybur et al., 2015b). These findings suggest that pathogen avoidance motives might shape political ideology via sexual strategies (and their resulting consequences for attitudes toward abortion and contraception) rather than motivations to avoid contact with outgroups. A recent multi-national study that examined the relation between pathogen DS and multiple dimensions of ideology offers another reason to reevaluate the outgroup-avoidance interpretation of pathogen DS and ideology (Tybur et al., 2016 ). Across 30 nations, the relation between pathogen DS and traditionalism - a dimension of ideology especially related to intragroup attitudes and norm adherence - is stronger than the relation between pathogen DS and social dominance orientation - a dimension of ideology especially related to intergroup attitudes and maintaining barriers between groups. These findings are also 
not straightforwardly consistent with the hypothesis that any relations between pathogen DS and ideology are based on the prophylactic benefits of avoiding contact with outgroups. Rather, they suggest that norm adherence aspects of ideology that might have anti-pathogen properties are appealing to high pathogen DS individuals. Candidate norms include sexual monogamy (relative to promiscuity), hygiene rituals, and food preparation techniques (see also Schaller and Murray, 2008). Ultimately, considerations of the dimensionality and strategic costs and benefits of DS have led to a healthy generation of competing hypotheses, many of which remain to be tested. DS and Psychopathology

Over the past two decades, researchers have found that pathogen DS relates to a number of psychopathologies, including small-animal phobias (De Jong and Merckelbach, 1998; Matchett and Davey, 1991), blood-injection-injury phobia (Sawchuk et al., 2000), obsessivecompulsive disorder (OCD; Olatunji et al., 2007 ), and eating disorders (Troop et al., 2002). Other methodologies complement individual differences approaches in suggesting that attention to disgust can advance understanding of some anxiety disorders (see Cisler et al., 2009 for an overview). For example, spider-phobic children report greater disgust toward spiders relative to healthy controls (De Jong et al., 1997), and children who receive disgust-related information about unfamiliar animals later endorse more fear beliefs toward those animals than children in the control group (Muris et al., 2008).

Many of the disorders linked to disgust involve anxieties toward situations or stimuli that are especially pathogen-risky: small animals, such as rodents and insects, frequently carry pathogens (Curtis and Biran, 2001); skin punctures create a breech through the skin, which otherwise acts as a pathogen-repelling armor; and contact with surfaces risks acquiring pathogens transmitted by others. These considerations have not been lost on clinical researchers, 
who have sometimes shaped hypotheses around 'disease avoidance' perspectives. Indeed, some models suggest that DS leads to a conscious overestimate of pathogen presence, which can in turn increase vigilance toward pathogens, which can in turn reinforce the estimates of pathogen presence (e.g., Widen and Olatunji, 2016). According to these models, high DS individuals are especially susceptible to psychopathologies that involve hypervigilance toward perceived pathogen presence - for example, contamination-related OCD.

Further understanding the tradeoffs underlying DS may allow us to gain a better understanding of topics such as ideology and psychopathology. However, considerations of tradeoffs alone do not speak to the sources of variability in DS. That is, they do not address the features of the environment or individual that might steer development toward a lower or higher DS. A handful of studies have begun to examine these sources of variation, sometimes with surprising results.

\section{What Causes Variation in DS?}

Some acts and objects, such as bodily wastes and sibling incest, elicit disgust across cultures (Curtis and Biran, 2001; Haidt et al., 1997). Nevertheless, cross-cultural differences in disgust elicitors are striking. For example, canines elicit disgust as a food source in the United States, but they are a delicacy in China. Nations differ markedly in their openness to casual sex (Schmitt, 2005) and, presumably, their disgust toward casual sex. And the targets of moral condemnation vary across cultures (Haidt et al., 1993; Haidt et al., 1997). Disgust also seems 'absent' at young ages, with children not avoiding prototype pathogen cues (e.g., feces) until after age two and not expressing disgust until after age five (see Rottman, 2014 for an overview). Together, these observations have been interpreted as suggesting that DS is largely shaped by social learning and, specifically, through parental modeling (Kim et al., 2013; Rozin et al., 2008; 
Widen and Olatunji, 2016; see also Siegal et al., 2011). Investigations of DS similarity within families have been taken as support for a parental transmission perspective - parent DS correlates strongly with offspring DS (Davey et al., 1993) and DS is also strongly correlated within sibling pairs (Rozin and Millman, 1987). However, a recent behavioral genetics study casts doubt on the parental transmission perspective. In a sample of over 500 twin pairs, Sherlock et al. (2016) found that DS is shaped by both environmental and genetic factors. However, any similarity between twins was caused by their shared genes rather than their shared environment, which would include parental modeling of trait DS (cf. Widen and Olatunji, 2016). Although environmental factors shaped about half of the variation in DS, those environmental factors were unshared by twins.

Of course, the fact that shared genes rather than shared environment underlie withinfamily similarity in DS is compatible with both cross-cultural variations in disgust elicitors and the observed developmental trajectory of disgust. As is the case with many individual differences variables, any socially transmitted aspects of DS could be shaped by peers (or other conspecifics) rather than by parents (Pinker, 2002). Further, nothing in the behavioral genetics data rules out the possibility that sources of pathogens (e.g., a hand that is normatively used in ablutions) are socially learned, either via parents or via peers, even if the intensity of disgust responses toward those sources (i.e., DS) is not modeled after parents' DS. The developmental timing of disgust is also compatible with behavioral genetics data. Indeed, many heritable traits minimally influenced by social learning (e.g., breasts and beards) are not present at birth, but instead reliably develop later in life (Tooby and Cosmides, 1992).

With parental modeling ruled out as a likely influence on DS, future research can focus on testing other potential sources of variation. Infection history could be one such source. 
Indeed, to the extent to which infection history is influenced by heritable immune function or exposure to pathogens, it could account for both genetic and unshared environment components of DS. However, existing research, while limited in size and scope, has not supported this hypothesis. In a study of 284 individuals living and raised in Bangladesh - a pathogen-rich nation - both recent and childhood infection history were unrelated to pathogen DS in adulthood (de Barra et al., 2014). Further, the cross-cultural study on ideology and disgust found that, across 30 nations, national infectious disease burden was unrelated to average within-nation pathogen DS (Tybur et al., 2016 ). Of course, the tradeoff perspective described earlier implies that pathogen DS should be calibrated based only on the costs that individuals pay in the event of infection, but also on the costs of avoiding pathogen cues. In pathogen-rich environments, avoidance of cues to pathogens might do little to mitigate infection risk. After all, if pathogens are in the water and readily spread by animal vectors (e.g., mosquitoes), then high investments in avoidance may not prevent infection, but they can certainly limit reproductive, social, and nutritional opportunities. Ultimately, though, these studies have used coarse assessments of infection history and ecological pathogen presence. More work is needed to adequately test how genetic and environmental factors related to infection relate to DS. And, of course, similar progress is needed in generating and testing hypotheses related to sexual and moral DS.

\section{Concluding Thoughts}

Researchers have been excited to find that DS relates to phenomena such as political ideology and psychopathology. Such findings promise to provide novel understandings of why individuals adopt liberal vs. conservative political positions, or why they experience potentially debilitating anxieties about washing and cleansing. The potential knowledge gleaned from these studies is constrained by our understanding of DS itself, though. Much recent progress has been 
made in understanding disgust as an emotion, and in understanding the dimensionality of DS, relation with personality, and heritability. Every slice of new information about DS can raise further questions. We now know that DS is heritable, and that parental modeling does not appear to shape DS. But what are the genetic and environmental sources shaping DS? Pathogen DS appears to relate to behavioral avoidance of pathogen cues, but perhaps not facial responses to pathogen cues. Do null relations with facial response reflect a disjunction between experienced and expressed disgust, or do they reflect underpowered studies? And, further, what differentiates pathogen DS from other variables used in the behavioral immune system literature, and what differentiates sexual DS from sociosexuality? Questions like these suggest that DS research has entered a second generation of sorts - it has matured from a new venture that shows promise for understanding a variety of phenomena to a multidisciplinary undertaking dedicated to understanding dimensionality, development, and cross-cultural variation. We hope that this chapter has sketched out promising directions for this second generation of research, and that future overviews of DS can provide answers to some of the questions posed. 


\section{Box 1}

\section{Disgust Propensity and Disgust Sensitivity}

Researchers have recently used the term "disgust propensity" to describe the constructs measured by the Disgust Scale and the Three Domain Disgust Scale. This shift in terminology stems from calls for distinctions between the frequency of experiencing disgust (disgust propensity) and the distress caused by experiencing disgust (disgust sensitivity; Van Overveld, De Jong, Peters, Cavanagh, \& Davey, 2006). Such calls followed observations that the content of disgust instruments overlaps with symptoms of specific psychopathologies (e.g., spider phobia, blood-injury phobia), and method variance might thus lead to an overestimation of the relation between trait disgust and psychopathology (Olatunji, Cisler, Deacon, Connolly, \& Lohr, 2007; Tolin, Woods, \& Abramowitz, 2006; Van Overveld et al., 2006). To address these concerns, researchers developed the Disgust Propensity and Sensitivity Scale (DPSS; Van Overveld et al., 2006), which aims to assess disgust propensity and disgust sensitivity without reference to specific disgust elicitors. The propensity items ask participants to indicate the frequency and intensity with which they, in general, experience disgust (e.g., "I experience disgust," "When I experience disgust, it is an intense feeling"), and the sensitivity items ask participants to indicate how unpleasant they find the experience of disgust (e.g., "It scares me when I feel nauseous," "When I feel disgusted, I worry that I might pass out").

We admit some skepticism toward this distinction (at least as it is currently made in the DPSS), for a few reasons. First, DPSS disgust sensitivity items might indirectly assess disgust propensity. For instance, answers to the disgust sensitivity item "When I feel disgusted, I worry that I might pass out" partly depend on how disgusted the individual imagines feeling - which is contingent on the respondent's disgust proneness. Further, because the negative appraisal of a 
disgust experience (i.e., disgust sensitivity) is measured in frequency, answers to these items partly depend on how often one experiences disgust in the first place (i.e., disgust propensity). Second, some disgust sensitivity items in the DPSS appear peripheral to disgust (e.g. "It scares me when I feel faint"). Third, even proponents of the distinction between disgust propensity and sensitivity use the terms interchangeably within the same article (e.g., Olatunji, Cisler, McKay, \& Phillips, 2010; Olatunji, Ebesutani, \& Reise, 2015).

Despite these issues, some studies suggest that the DPSS is useful in assessing the role of disgust in psychopathologies, and that the propensity and sensitivity subscales are uniquely related to different psychopathological symptoms (Cisler, Olatunji, \& Lohr, 2009; Olatunji et al., 2007; Van Overveld et al., 2006). However, nonclinical participants tend to endorse minimal agreement with majority of the DPSS items - especially the sensitivity items - suggesting that the DPSS insufficiently captures nonclinical participants' experience of disgust (Olatunji et al., 2007). Other instruments may therefore be more useful for assessing disgust tendencies in the general population.

Ultimately, for this chapter, we use the term "disgust sensitivity" (DS) to refer to what some authors have recently described as "disgust propensity" or "disgust proneness." 


\section{Box 2}

\section{Animal Reminders}

In developing the Disgust Scale, Haidt and colleagues (1994) categorized some of their proposed disgust domains - namely, hygiene, body envelope violations, sex, and death - under a broader umbrella of "animal reminder" disgust. Using a perspective similar to Terror Management Theory (Goldenberg, Pyszczynski, Greenberg, \& Solomon, 2000), they suggested that humans have a fundamental need to avoid being reminded that they are animals and, hence, mortal (see also Rozin et al., 2008b). According to this perspective, anything that reminds us that we are animals violates this need and can activate existential anxiety, and disgust toward animal reminders relieves this anxiety. Despite the large volume of references to animal reminder disgust in the literature, though, three observations cast serious doubt on the assumptions underlying the animal reminder framework and on the validity of the animal reminder taxonomy (for lengthier summaries, see Kollareth \& Russell, in press-b; Royzman \& Sabini, 2001; Tybur et al., 2009, 2013).

First, no evidence indicates that experiencing disgust decreases the degree to which humans recognize their nature as animals or their own mortality. Similarly, no evidence indicates that experiencing disgust palliates any unpleasant feelings that accompany reminders of animality or mortality.

Second, most features of animal morphology that overlap with human morphology (e.g., having two eyes, having four limbs) - and hence could "remind" humans that we are animals - do not elicit disgust. The same goes for most animal behaviors that overlap with human behaviors (e.g., breathing, sleeping, childcare). Even going to efforts to remind participants that they are animals does not elicit disgust (Kollareth \& Russell, in press-b). Although some disgust elicitors 
relate to sex, hygiene, death, and envelope violations, no evidence suggests that they elicit disgust because they remind us that we are animals.

Third, individual differences data speak against the existence of the animal reminder domain described by Haidt and colleagues (1994), who argue that, whereas disgust toward food, body products, and animals serves an anti-pathogen function, disgust toward sex, poor hygiene, envelope violations, and death functions to neutralize reminders that we are animals. In creating the Disgust Scale-Revised, Olatunji and colleagues (2007) find that sex and hygiene items do not load on the same factor as the death and envelope violation items, which were combined and labeled as an animal reminder factor. Similarly, Tybur and colleagues (2009) find that the animal reminder factor of the Disgust Scale-Revised was unrelated to sexual disgust, independent of pathogen disgust. 


\section{Table 1}

Correlations between TDDS factors and NEO PI-3 (left of slash) and HEXACO PI-R (right of slash) personality factors. Values are based on Tybur et al. (2011) and Tybur \& De Vries (2013) (respectively). Note that correlations between TDDS and HEXACO factors differ slightly from those presented by Tybur and De Vries (2013). The correlations reported here control for participant sex and age, whereas those reported by Tybur and De Vries did not.

\begin{tabular}{lccc}
\hline $\begin{array}{l}\text { NEO PI-3/HEXACO PI-R } \\
\text { factor }\end{array}$ & \multicolumn{3}{c}{ TDDS factor } \\
\cline { 2 - 4 } & Pathogen & Sexual & Moral \\
\hline Neuroticism/Emotionality & $.10 / .18$ & $.03 / .10$ & $-.13 / .02$ \\
Extraversion & $-.05 / .01$ & $-.02 /-.12$ & $.07 / .06$ \\
Openness & $-.24 /-.11$ & $-.38 /-.08$ & $-.03 / .00$ \\
Agreeableness & $-.13 /-.17$ & $.18 / .09$ & $.25 / .05$ \\
Conscientiousness & $.02 / .11$ & $.11 / .11$ & $.28 / .26$ \\
Honesty-Humility & $--/-.08$ & $--/ .17$ & $--/ .29$ \\
\hline
\end{tabular}


Figure 1

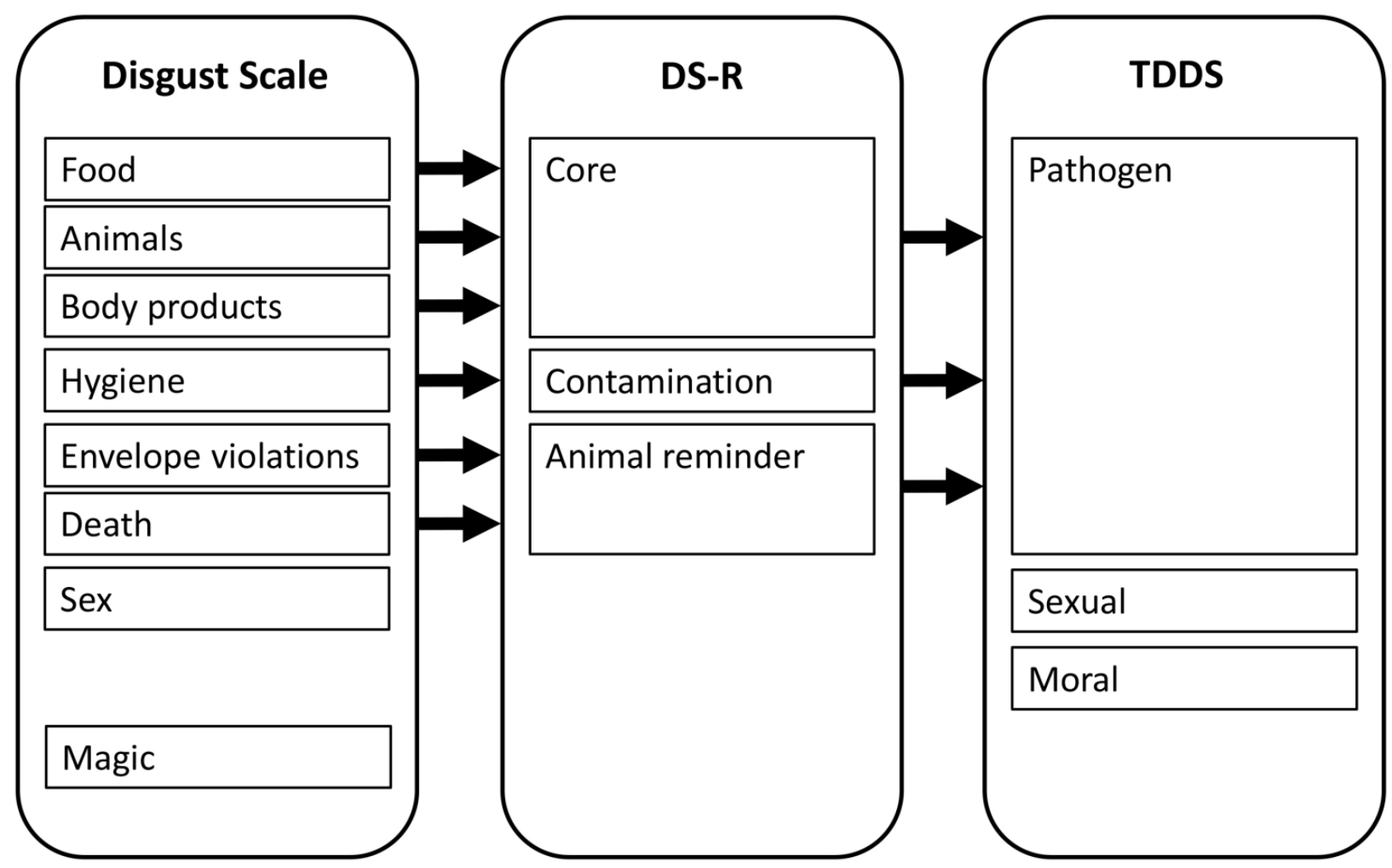

The original, 32-item Disgust Scale (Haidt et al., 1994) was designed to measure - and was typically analyzed as having - eight factors: Food, Animals, Body Products, Hygiene, Envelope Violations, Death, Sex, and Magic. The revision of the Disgust Scale, the DS-R (Olatunji et al., 2007) reduced the Disgust Scale to 25 items, and it has been interpreted as measuring three highly correlated factors. The Core factor included items from the Disgust Scale Food, Animals, and Body Products factors; the Contamination factor included items from the Disgust Scale Hygiene factor; and the Animal Reminder factor included items from the Disgust Scale Envelope Violations and Death factors (note that items on the Magic factor were incorporated across multiple DS-R factors). In the 21-item Three Domain Disgust Scale (Tybur et al., 2009), those three highly correlated factors - and most of the content of the Disgust Scale - are subsumed 
under a single "Pathogen" factor. The TDDS also includes Sexual and Moral factors, which are largely unmeasured by the Disgust Scale and the DS-R. 


\section{References}

Al-Shawaf, L., Lewis, D. M., \& Buss, D. M. (2015). Disgust and mating strategy. Evolution and Human Behavior, 36, 199-205.

Allport, G. W., \& Odbert, H. S. (1936). Trait-names: A psycholexical study. Psychological Monographs, 47, Whole No. 211.

Aarøe, L., Osmundsen, M., \& Petersen, M. B. (2016). Distrust as a disease avoidance strategy: Individual differences in disgust sensitivity regulate generalized social trust. Frontiers in Psychology, 7, 1-14.

Ashton, M. C., \& Lee, K. (2009). The HEXACO-60: A short measure of the major dimensions of personality. Journal of Personality Assessment, 91, 340-345.

Ashton, M. C., Lee, K., \& De Vries, R. E. (2014). The HEXACO honesty-humility, agreeableness, and emotionality factors: A review of research and theory. Personality and Social Psychology Review, 18, 139-152.

Borg, C., \& De Jong, P. J. (2012). Feelings of disgust and disgust-induced avoidance weaken following induced sexual arousal in women. PLoS ONE, 7, e44111

Bourdage, J. S., Lee, K., Ashton, M. C., \& Perry, A. (2007). Big Five and HEXACO model personality correlates of sexuality. Personality and Individual Differences, 43, 1506-1516.

Case, T. I., Repacholi, B. M., \& Stevenson, R. J. (2006). My baby doesn't smell as bad as yours: The plasticity of disgust. Evolution and Human Behavior, 27, 357-365.

Chapman, H. A., \& Anderson, A. K. (2013). Things rank and gross in nature: A review and synthesis of moral disgust. Psychological Bulletin, 139, 300-327.

Chapman, H. A., \& Anderson, A. K. (2014). Trait physical disgust is related to moral judgments outside of the purity domain. Emotion, 14, 341-348. 
Cisler, J. M., Olatunji, B. O., \& Lohr, J. M. (2009). Disgust, fear, and the anxiety disorders: A critical review. Clinical Psychology Review, 29, 34-46.

Credé, M., Harms, P., Niehorster, S., \& Gaye-Valentine, A. (2012). An evaluation of the consequences of using short measures of the Big Five personality traits. Journal of Personality and Social Psychology, 102, 874-888.

Curtis, V., \& Biran, A. (2001). Dirt, disgust, and disease: Is hygiene in our genes? Perspectives in Biology and Medicine, 44, 17-31

Davey, G. C. L., Forster, L., \& Mayhew, G. (1993). Familial resemblances in disgust sensitivity and animal phobias. Behaviour Research and Therapy, 31, 41-50.

Deacon, B., \& Olatunji, B. O. (2007). Specificity of disgust sensitivity in the prediction of behavioral avoidance in contamination fear. Behaviour Research and Therapy, 45, 21102120.

De Barra, M., Islam, M. S., \& Curtis, V. (2014). Disgust sensitivity is not associated with health in a rural Bangladeshi sample. PloS one, 9, e100444.

De Jong, P. J., Andrea, H., \& Muris, P. (1997). Spider phobia in children: Disgust and fear before and after treatment. Behaviour Research and Therapy, 35, 559-562.

De Jong, P. J., \& Merckelbach, H. (1998). Blood-injection-injury phobia and fear of spiders: Domain specific individual differences in disgust sensitivity. Personality and Individual Differences, 24, 153-158.

De Jong, P. J., Van Overveld, M., \& Peters, M. L. (2011). Sympathetic and parasympathetic responses to a core disgust video clip as a function of disgust propensity and disgust sensitivity. Biological Psychology, 88, 174-179. 
De Vries, R. E. (2013). The 24-item brief HEXACO inventory (BHI). Journal of Research in Personality, 47, 871-880.

Druschel, B. A., \& Sherman, M. F. (1999). Disgust sensitivity as a function of the Big Five and gender. Personality and Individual Differences, 26, 739-748.

Duncan, L. A., Schaller, M., \& Park, J. H. (2009). Perceived vulnerability to disease:

Development and validation of a 15-item self-report instrument. Personality and Individual differences, 47, 541-546.

Fan, Q., \& Olatunji, B. O. (2013). Individual differences in disgust sensitivity and health-related avoidance: Examination of specific associations. Personality and Individual Differences, $55,454-458$.

Fessler, D. M., Arguello, A. P., Mekdara, J. M., \& Macias, R. (2003). Disgust sensitivity and meat consumption: A test of an emotivist account of moral vegetarianism. Appetite, 41, 3141.

Fincher, C. L., \& Thornhill, R. (2012). Parasite-stress promotes in-group assortative sociality: The cases of strong family ties and heightened religiosity. Behavioral and Brain Sciences, 35, 1-59.

Fridlund, A. J. (1991). Sociality of social smiling: Potentiation by an implicit audience. Journal of Personality and Social Psychology, 60, 229-240.

Gangestad, S. W., \& Grebe, N. M. (2014). Pathogen avoidance within an integrated immune system: Multiple components with distinct costs and benefits. Evolutionary Behavioral Sciences, 8, 226-234.

Goldberg, L. R. (1990). An alternative "description of personality": The Big-Five factor structure. Journal of Personality and Social Psychology, 59, 1216-1229. 
Goldenberg, J. L., Pyszczynski, T., Greenberg, J., \& Solomon, S. (2000). Fleeing the body: A terror management perspective on the problem of human corporeality. Personality and Social Psychology Review, 4, 200-218.

Goldenberg, J. L., Pyszczynski, T., Greenberg, J., Solomon, S., Kluck, B., \& Cornwell, R. (2001). I am not an animal: Mortality salience, disgust, and the denial of human creatureliness. Journal of Experimental Psychology: General, 130, 427-435.

Gray, K., \& Keeney, J. E. (2015). Impure or just weird? Scenario sampling bias raises questions about the foundation of morality. Social Psychological and Personality Science, 6, 859868.

Haidt, J., Koller, S. H., \& Dias, M. G. (1993). Affect, culture, and morality, or is it wrong to eat your dog? Journal of Personality and Social Psychology, 65, 613-628.

Haidt, J., McCauley, C., \& Rozin, P. (1994). Individual differences in sensitivity to disgust: A scale sampling seven domains of disgust elicitors. Personality and Individual Differences, $16,701-713$.

Haidt, J., Rozin, P., McCauley, C., \& Imada, S. (1997). Body, psyche, and culture: The relation of disgust to morality. Psychology and Developing Societies, 9, 107-131.

Harmon-Jones, E., \& Peterson, C. K. (2008). Effect of trait and state approach motivation on aggression. Journal of Research in Personality, 42, 1381-1385

Hennig, J., Possel, P., \& Netter, P. (1996). Sensitivity to disgust as a predictor of neuroticism: A psychobiological approach. Personality and Individual Differences, 20, 589-596

Hertenstein, M. J., Keltner, D., App, B., Bulleit, B. A., \& Jaskolka, A. R. (2006). Touch communicates distinct emotions. Emotion, 6, $528-533$ 
Hoefling, A., Likowski, K. U., Deutsch, R., Häfner, M., Seibt, B., Mühlberger, A.,... Strack, F. (2009). When hunger finds no fault with moldy corn: Food deprivation reduces foodrelated disgust. Emotion, 9, 50-58.

Inbar, Y., \& Pizarro, D. A. (2016). Pathogens and politics: Current research and new questions. Social and Personality Psychology Compass, 10, 365-374.

Inbar, Y., Pizarro, D., Iyer, R., \& Haidt, J. (2012). Disgust sensitivity, political conservatism, and voting. Social Psychological and Personality Science, 3, 537-544.

Jonason, P. K., Li, N. P., Webster, G. D., \& Schmitt, D. P. (2009). The dark triad: Facilitating a short-term mating strategy in men. European Journal of Personality, 23, 5-18.

Kim, E. H., Ebesutani, C., Young, J., \& Olatunji, B. O. (2013). Factor structure of the disgust scale-revised in an adolescent sample. Assessment, 20, 620-631.

Kollareth, D., \& Russell, J. A. (in press-a). The English word disgust has no exact translation in Hindi or Malayalam. Cognition and Emotion.

Kollareth, D., \& Russell, J. A. (in press-b). Is it disgusting to be reminded that you are an animal? Cognition and Emotion.

Kurzban, R., Dukes, A., \& Weeden, J. (2010). Sex, drugs and moral goals: Reproductive strategies and views about recreational drugs. Proceedings of the Royal Society B:

Biological Sciences, 277, 3501-3508

Matchett, G., \& Davey, G. C. (1991). A test of a disease-avoidance model of animal phobias. Behaviour Research and Therapy, 29, 91-94.

McCrae, R. R., Costa, P. T., \& Martin, T. A. (2005). The NEO-PI-3: A more readable revised NEO personality inventory. Journal of Personality Assessment, 84, 261-270. 
Muris, P., Mayer, B., Huijding, J., \& Konings, T. (2008). A dirty animal is a scary animal! Effects of disgust-related information on fear beliefs in children. Behaviour Research and Therapy, 46, 137-144.

Olatunji, B. O., Adams, T., Ciesielski, B., David, B., Sarawgi, S., \& Broman-Fulks, J. (2012). The three domains of disgust scale factor structure, psychometric properties, and conceptual limitations. Assessment, 19, 205-225.

Olatunji, B. O., Cisler, J. M., Deacon, B. J., Connolly, K., \& Lohr, J. M. (2007). The Disgust Propensity and Sensitivity Scale-Revised: Psychometric properties and specificity in relation to anxiety disorder symptoms. Journal of Anxiety Disorders, 21, 918-930.

Olatunji, B. O., Cisler, J., McKay, D., \& Phillips, M. L. (2010). Is disgust associated with psychopathology? Emerging research in the anxiety disorders. Psychiatry Research, 175, $1-10$.

Olatunji, B. O., Ebesutani, C., \& Reise, S. P. (2015). A bifactor model of disgust proneness: Examination of the Disgust Emotion Scale. Assessment, 22, 248-262

Olatunji, B. O., Lohr, J. M., Sawchuk, C. N., \& Tolin, D. F. (2007). Multimodal assessment of disgust in contamination-related obsessive-compulsive disorder. Behaviour Research and Therapy, 45, 263-276.

Olatunji, B. O., Haidt, J., McKay, D., \& David, B. (2008). Core, animal reminder, and contamination disgust: Three kinds of disgust with distinct personality, behavioral, physiological, and clinical correlates. Journal of Research in Personality, 42, 1243-1259.

Olatunji, B. O., Tomarken, A., \& Puncochar, B. D. (2013). Disgust propensity potentiates evaluative learning of aversion. Emotion, 13, 881-890. 
Olatunji, B. O., \& Sawchuk, C. N. (2005). Disgust: Characteristic features, social manifestations, and clinical implications. Journal of Social and Clinical Psychology, 24, 932-962.

Olatunji, B. O., Tomarken, A., \& Puncochar, B. D. (2013). Disgust propensity potentiates evaluative learning of aversion, Emotion, 13, 881-890.

Olatunji, B. O., Williams, N. L., Tolin, D. F., Sawchuk, C. N., Abramowitz, J. S., Lohr, J. M., \& Elwood, L. (2007). The Disgust Scale: Item analysis, factor structure, and suggestions for refinement. Psychological Assessment, 19, 281-297.

Park, J. H. (2015). Introversion and human-contaminant disgust sensitivity predict personal space. Personality and Individual Differences, 82, 185-187.

Phillips, M. L., Fahy, T., David, A. S., \& Senior, C. (1998). Disgust: The forgotten emotion of psychiatry. British Journal of Psychiatry, 172, 373-375.

Pinker, S. (2002). The blank slate: The modern denial of human nature. New York, NY: Penguin Putnam.

Pond, R. S., DeWall, C. N., Lambert, N. M., Deckman, T., Bonser, I. M., \& Fincham, F. D. (2012). Repulsed by violence: Disgust sensitivity buffers trait, behavioral, and daily aggression. Journal of Personality and Social Psychology, 102, 175-188.

Quintelier, K. J., Ishii, K., Weeden, J., Kurzban, R., \& Braeckman, J. (2013). Individual differences in reproductive strategy are related to views about recreational drug use in Belgium, the Netherlands, and Japan. Human Nature, 24, 196-217

Reynolds, L. M., McCambridge, S. A., Bissett, I. P., \& Consedine, N. S. (2014). Trait and state disgust: An experimental investigation of disgust and avoidance in colorectal cancer decision scenarios. Health Psychology, 33, 1495-1506. 
Roseman, I. J., Wiest, C., \& Swartz, T. S. (1994). Phenomenology, behaviors, and goals differentiate discrete emotions. Journal of Personality and Social Psychology, 67, 206-221.

Rottman, J. (2014). Evolution, development, and the emergence of disgust. Evolutionary Psychology, 12, 417-433.

Royzman, E., \& Sabini, J. (2001). Something it takes to be an emotion: The interesting case of disgust. Journal for the Theory of Social Behavior, 31, 29-59.

Rozin, P., Haidt, J., \& McCauley, C. R. (2009). Disgust: The body and soul emotion in the 21st century. In B. O. Olatunji \& D. McKay (Eds.), Disgust and its disorders (pp. 9-29). Washington, DC: American Psychological Association.

Rozin, P., Haidt, J., \& McCauley, C. R. (2008). Disgust. In M. Lewis, J. M. Haviland-Jones, \& L. F. Barrett (Eds.), Handbook of emotions (3rd ed., pp. 757-776). New York, NY: Guilford Press

Rozin, P., Haidt, J., McCauley, C., Dunlop, L., \& Ashmore, M. (1999). Individual differences in disgust sensitivity: Comparisons and evaluations of paper-and-pencil versus behavioral measures. Journal of Research in Personality, 33, 330-351.

Rozin, P., \& Fallon, A E. (1980). Psychological categorization of foods and non-foods: A preliminary taxonomy of food rejections Appetite, 1, 193-201.

Rozin, P., Fallon, A. E., \& Mandell, R. (1984). Family resemblance in attitudes to food. Developmental Psychology, 20, 309-314.

Rozin, P., \& Millman, L. (1987). Family environment, not heredity, accounts for family resemblances in food preferences and attitudes: A twin study. Appetite, 8, 125-134. 
Rozin, P., Taylor, C., Ross, L., Bennett, G., \& Hejmadi, A. (2005). General and specific abilities to recognize negative emotions, especially disgust, as portrayed in the face and the body. Cognition \& Emotion, 19, 397-412.

Sawchuk, C. N., Lohr, J. M., Tolin, D. F., Lee, T. C., \& Kleinknecht, R. A. (2000). Disgust sensitivity and contamination fears in spider and blood-injection-injury phobias. Behaviour Research and Therapy, 38, 753-762.

Schaller, M., \& Murray, D. R. (2008). Pathogens, personality and culture: Disease prevalence predicts worldwide variability in sociosexuality, extraversion, and openness to experience. Journal of Personality and Social Psychology, 95, 212-221.

Schmitt, D. P. (2005). Sociosexuality from Argentina to Zimbabwe: A 48-nation study of sex, culture, and strategies of human mating. Behavioral and Brain Sciences, 28, 247-275.

Sherlock, J. M., Zietsch, B. P., Tybur, J. M., \& Jern, P. (2016). The quantitative genetics of disgust sensitivity. Emotion, 16, 43-51.

Sherman, G. D., Haidt, J., \& Clore, G. L. (2012). The faintest speck of dirt disgust enhances the detection of impurity. Psychological Science, 23, 1506-1514.

Shook, N. J., Terrizzi, J. A., Clay, R., \& Oosterhoff, B. (2015). In defense of pathogen disgust and disease avoidance: A response to Tybur et al. (2015). Evolution and Human Behavior, 36, 498-502.

Siegal, M., Fadda, R., \& Overton, P. G. (2011). Contamination sensitivity and the development of disease-avoidant behaviour. Philosophical Transactions of the Royal Society of London Series B, Biological Sciences, 366, 3427-3432 
Simpson, J. A., \& Gangestad, S. W. (1991). Individual differences in sociosexuality: Evidence for convergent and discriminant validity. Journal of Personality and Social Psychology, $60,870-883$.

Stark, R., Walter, B., Schienle, A., \& Vaitl, D. (2005). Psychophysiological correlates of disgust and disgust sensitivity. Journal of Psychophysiology, 19, 50-60.

Susskind, J. M., Lee, D. H., Cusi, A., Feiman, R., Grabski, W., \& Anderson, A. K. (2008). Expressing fear enhances sensory acquisition. Nature Neuroscience, 11, 843-850.

Terrizzi, J. A., Shook, N. J., \& McDaniel, M. A. (2013). The behavioral immune system and social conservatism: A meta-analysis. Evolution and Human Behavior, 34, 99-108.

Tolin, D. F., Woods, C. M., \& Abramowitz, J. S. (2006). Disgust sensitivity and obsessivecompulsive symptoms in a non-clinical sample. Journal of Behavior Therapy and Experimental Psychiatry, 37, 30-40.

Tooby, J. \& Cosmides, L. (1992). The psychological foundations of culture. In J. Barkow, L. Cosmides, \& J. Tooby (Eds.), The adapted mind: Evolutionary psychology and the generation of culture (pp. 19-113). New York, NY: Oxford University Press.

Troop, N. A., Treasure, J. L., \& Serpell, L. (2002). A further exploration of disgust in eating disorders. European Eating Disorders Review, 10, 218-226.

Tybur, J. M., Bryan, A. D., Lieberman, D., Caldwell Hooper, A. E., \& Merriman, L. A. (2011). Sex differences and sex similarities in disgust sensitivity. Personality and Individual Differences, 51, 343-348.

Tybur, J. M., \& De Vries, R. E. (2013). Disgust sensitivity and the HEXACO model of personality. Personality and Individual Differences, 55, 660-665. 
Tybur, J. M., Frankenhuis, W. E., \& Pollet, T. V. (2014). Behavioral immune system methods: Surveying the present to shape the future. Evolutionary Behavioral Sciences, 8, 274-283.

Tybur, J. M., Inbar, Y., Aarøe, L., Barclay, P., Barlow, F. K., de Barra, M.,... Žeželj, I. (in press). Parasite stress and pathogen avoidance relate to distinct dimensions of political ideology across 30 nations. Proceedings of the National Academy of Sciences, 113, 1240812413.

Tybur, J. M., Inbar, Y., Güler, E., \& Molho, C. (2015a). Is the relation between pathogen avoidance and ideological conservatism explained by sexual strategies? Evolution and Human Behavior, 36, 489-497.

Tybur, J. M., Inbar, Y., Güler, E., \& Molho, C. (2015b). Pathogen disgust requires no defense: a response to Shook, Terrizzi, Clay, \& Oosterhoff (2015). Evolution and Human Behavior, $36,502-504$.

Tybur, J. M., \& Lieberman, D. (2016). Human pathogen avoidance adaptations. Current Opinion in Psychology, 7, 6-11.

Tybur, J. M., Lieberman, D., \& Griskevicius, V. (2009). Microbes, mating, and morality: Individual differences in three functional domains of disgust. Journal of Personality and Social Psychology, 97, 103-122.

Tybur, J. M., Lieberman, D., Kurzban, R., \& DeScioli, P. (2013). Disgust: Evolved function and structure. Psychological Review, 120, 65-84.

Tybur, J. M., Merriman, L. A., Caldwell, A. E., McDonald, M. M., \& Navarrete, C. D. (2010). Extending the behavioral immune system to political psychology: Are political conservativism and disgust sensitivity really related? Evolutionary Psychology, 8, 599616. 
Van Overveld, M., De Jong, P. J., \& Peters, M. L. (2010). The disgust propensity and sensitivity scale-revised: its predictive value for avoidance behavior. Personality and Individual Differences, 49, 706-711.

Van Overveld, W. J. M., De Jong, P. D., Peters, M. L., Cavanagh, K., \& Davey, G. C. L. (2006). Disgust propensity and disgust sensitivity: Separate constructs that are differentially related to specific fears. Personality and Individual Differences, 41, 1241-1252.

Walls, M. M., \& Kleinknect, R. A. (1996, April). Disgust factors as predictors of blood-injury fear and fainting. Paper presented at the annual meeting of the Western Psychological Association, San Jose, CA.

Widen, S. C., \& Olatunji, B. O. (2016). A developmental perspective on disgust: Implications for obsessive-compulsive disorder. Current Behavioral Neuroscience Reports, 3, 204-210.

Wilson, G. D., Kumari, V., Gray, J. A., \& Corr, P. J. (2000). The role of neuroticism in startle reactions to fearful and disgusting stimuli. Personality and Individual Differences, 29, 1077-108. 